\title{
Contrasting effects of repetition across tasks: Implications for understanding the nature of refractory behavior and models of semantic memory
}

\author{
EMER M. E. Forde ANd GLYN W. HuMphreys \\ University of Birmingham, Birmingham, England
}

\begin{abstract}
We describe a patient (J.M.) who showed "refractory" behavior in picture-word matching tasks - that is, his performance became poorer when items were repeated. This contrasts with the facilitatory effects of repetition usually observed in normal participants. We show for the first time that there can be facilitatory effects of repetition on some tasks, even though refractory behavior is shown on the same items in other tasks. In particular, in Experiments 1 and 2, we demonstrate that J.M. showed contrasting effects of repetition across different components of the language system: There were facilitatory effects of repetition priming on lexical decision but refractory behavior on picture-word matching. In Experiments 3 and 4, we demonstrate that J.M. showed contrasting effects of repetition within the same system (semantic memory). His performance became refractory when items were repeated in picture-word matching (Experiment 3), but it was facilitated when items were repeated in superordinate categorization (Experiment 4). These contrasting patterns of facilitation and interference from repetition priming have implications for understanding the nature of refractory behavior and for constraining theoretical accounts of semantic memory.
\end{abstract}

One standard finding in studies of human cognition is that our responses to stimuli are facilitated when items are repeated. This finding has been reported across a range of tasks, from perceptual identification of words and pictures (Durso \& Johnson, 1979; Jacoby \& Dallas, 1981; Morton, 1979), through to lexical decision (Salasoo, Shiffrin, \& Feustel, 1985) and deciding whether objects are possible or not (Schacter \& Cooper, 1993). Facilitatory effects of repetition occur both with brief intervals between stimuli and when stimuli are repeated even a week later (Jacoby \& Dallas, 1981). Such priming effects are often attributed either to prolonged activation of stimulus representations (Morton, 1979) or to weight changes affecting the connections between component parts of the representation of the stimulus (see, e.g., Burton, 1994, for one simulation).

In contrast to this standard, facilitatory effect of repetition on normal participants are data from neuropsychological patients who show so-called "refractory behavior."1 First described by Warrington and McCarthy (1983), patients showing refractory behavior have a worsening pattern of performance when items are repeated. In their original article, Warrington and McCarthy (1983) tested a global aphasic patient using picture-word matching tasks. The ability to point to a target picture among a set of distractors became less accurate when the target was repeated, relative to when it was probed for the first time. Similar data have subsequently been reported by Cipo- lotti, McCarthy, Warrington, and colleagues (Cipolotti \& Warrington, 1995; McNeil, Cipolotti, \& Warrington, 1994; Warrington \& Crutch, 2004; Warrington \& McCarthy, 1987) and by ourselves (Forde \& Humphreys, 1995, 1997). In each case, the negative effects of repetition increased when targets were surrounded by semantically related neighbors, and they increased with shorter intervals between the last response and the next stimulus presentation. The present article is concerned with the relations between the facilitatory effects of repetition often observed in normal participants and the negative effects apparent in "refractory" patients. In particular, we show for the first time that there can be facilitatory effects of repetition (and also of semantic priming) on some tasks, even though refractory behavior is demonstrated on other tasks. These contrasting patterns of facilitation and interference from priming help to constrain our understanding of refractory behavior and our understanding of the nature of our stored representations of stimuli.

Perhaps the most obvious account of refractory behavior is that the access procedures and/or representations for particular items become abnormally inhibited following stimulus presentation (see McCarthy \& Kartsounis, 2000). As a consequence, performance decreases when the stimulus is re-presented. Also, if there is a spread of inhibition between semantic neighbors, then presenting semantic distractors along with the target will exacer-

G.W. Humphreys, g.w.humphreys@bham.ac.uk 
bate the problem. A somewhat different view, though, is that refractory behavior occurs because items remain activated, rather than inhibited, for an abnormally long time (Forde \& Humphreys, 1997). Abnormally prolonged activation, especially if coupled with some degree of semantic impairment that makes it difficult to distinguish between close neighbors in semantic space, could lead to the observed pattern of worse performance when a patient is tested at Time 2 after earlier testing at Time 1. In studies of refractory behavior, a repeat response to a target is typically tested after at least one response has been made to the other items present. This preactivation of other items, if sustained to the time when the target is probed for a second time, would again generate competition for selection of the target's already weak representation, and this could even disrupt performance relative to when items are tested for a first time - the hallmark of refractory behavior. We examined these proposals in the present article. In Experiments 1 and 2, we assessed whether our patient, J.M., showed beneficial effects from repetition at one level of the language system (the orthographic input lexicon) even when he manifested deleterious effects of repetition, on the same items, in tasks requiring access to detailed semantic knowledge. Any evidence for facilitation would be consistent with there being persistent activation in at least some language representations. However, it is possible that there could be persistent activation at one level (e.g., an input lexicon) and inhibition at another (e.g., in semantic memory). In Experiments 3 and 4, we evaluated whether J.M. could show beneficial and deleterious effects of repetition within the same system (semantic memory) according to whether the task requires access to general or fine-grained information (superordinate categorization vs. picture-word matching). The data suggest that, at least for J.M., there is prolonged activation of stored representations not only at a lexical but also at a general semantic level. This evidence for persistent activation at a semantic level contradicts an inhibitory account of performance. We discuss the implications for understanding the nature of refractory deficits in patients.

\section{Access and Representation Impairments}

Data on different patterns of priming in refractory patients are also relevant to the distinction between so-called "access" and "degraded representation" impairments in neuropsychological patients. Patients with access impairments are held to have intact stored representations of stimuli, but they fail to access these representations on some occasions following their brain lesion. In contrast, patients with degraded representation can have intact access procedures, but fail tasks because they have lost the representations that would normally support performance. Shallice (1988) outlined a number of criteria to distinguish between these two patient groups at an empirical level. For example, he argued that patients with access deficits (1) should perform inconsistently across items over time, (2) should show little effect of item frequency, and (3) should find tasks difficult particularly under fast stimulus presentation conditions (when problems in access are stressed). In addition, the access deficit should (1) affect the retrieval of superordinate and subordinate information alike, because, once an item has been accessed (or fails to be accessed), all the information should be available (or unavailable, in the case of a failure in access), and (2) be reduced if the access process is facilitated by semantic priming. In contrast, he argued that a patient with a storage impairment should (1) show item-specific consistency over time, (2) be significantly better with high-frequency items, and (3) be better at accessing superordinate information in comparison with more detailed attributes. In addition, patients with degraded representations should not benefit from slower presentation rates or from semantic priming. Patients showing refractory behavior are usually thought to have an access deficit rather than a set of degraded representations for the affected items. For example, the patients are all inconsistent in their performance over time, and, as we noted above, they show effects of stimulus presentation rate and semantic relatedness (fast presentation rates and semantic distractors being bad for performance). Warrington and McCarthy (1983, 1987; see also Warrington \& Cipolotti, 1996) suggested that this constellation of deficits arose because damage led to access processes to semantics becoming temporarily (and abnormally) inhibited, following activation of a target item. They proposed that the patients were helped by long presentation intervals because such intervals allowed the target item to return to a baseline activation level. They also argued that the refractory state could spread along a semantic gradient to other categorically related items. This could account for patients' poorer performance with sets of related in comparison with unrelated items.

In previous work, we have also reported a case study of a patient with a similar pattern of performance to the other category-specific access dysphasics (Forde \& Humphreys, 1995, 1997). This patient, J.M., who is also the subject of the present study, was globally aphasic. In addition, J.M. (1) showed category-specific effects within the domain of proper names (see also Warrington \& McCarthy, 1987), (2) was inconsistent across items over time, (3) was worse with a short response-stimulus interval, (4) showed little effect of item frequency, but (5) he was worse when items were semantically related (see the Case Report for details). Critically, performance on matching tasks decreased when items were repeated, relative to when they were presented for the first time. We provided empirical support for the idea that the effects spread along a semantic gradient, because J.M.'s performance on auditory word-written word matching tasks was worse when initially probed items were replaced by new items that were related to the original targets, than when targets were replaced by unrelated items (Forde \& Humphreys, 1995).

Although the above results are consistent with an access impairment, Forde and Humphreys (1997) further demonstrated that the effect of repetition could spread across modalities (i.e., from words to pictures). This generalization across modalities is difficult to reconcile with a deficit in an access route to semantic memory, which should be specific to one input modality (though see Warrington \& Crutch, 2004). Instead, Forde and Humphreys (1997) proposed that the deficit lay within the semantic system itself. For 
example, semantic representations may become refractory immediately after being probed. The "spread" of refractoriness between related items may follow because semantic representations are distributed (see Rogers et al., 2004), so that related items suffer if common elements in their shared representation are inhibited. Once such a distributed representation becomes refractory, all items recognized on the basis of the affected representation are impaired. Alternatively, as noted previously, semantic representations may remain in an abnormally active state, blocking the selection of responses to stimuli within the affected set. If these arguments for a semantic locus to the deficit are correct, then doubt may be cast on the criteria for distinguishing between access and representational deficits in patients (see also Rapp \& Caramazza, 1993). We will discuss this in more detail in the General Discussion, following our presentation of new evidence on facilitatory effects of priming, as well as refractory effects, on J.M.'s performance of different tasks.

\section{Case Report}

J.M., a 72-year-old man, formerly head of languages at a grammar school, suffered a left hemisphere stroke in February 1992. A CAT scan showed a large hypodense area in the left temporoparietal region (see Forde \& Humphreys, 1995). Following his stroke, J.M. had right-sided hemiparesis, which left both his arm and leg weak. A year later, he had no voluntary control over his right arm but regained sufficient motor control over his leg to allow him to walk unaided. He scored 27/60 on Raven's Standard Progressive Matrices, which is intellectually average for his age. Prior to his stroke, J.M. was fluent in seven languages (Dutch, English, French, German, Italian, Spanish, and Russian). However, after the stroke, J.M. was globally aphasic, and his spoken output was limited to a single repetitive utterance ("da, da"). He had minimal written output for some single letters but for no words. He was able to write $15 / 26$ and 13/26 letters to dictation on Time 1 and Time 2, respectively.

J.M. performed relatively well on tests designed to tap access to stored modality-specific perceptual knowledge. For example, he scored 55/60 (92\%) correct on visual lexical decision and 51/60 (85\%) on auditory lexical decision (Psycholinguistic Assessment of Language Processing [PALPA] Tests 6 and 26; Kay, Lesser, \& Coltheart, 1992). On an object decision test from the Birmingham Object Recognition Battery (BORB; Riddoch \& Humphreys, 1993), he scored 32/32 (100\%). However, in contrast to this retained knowledge, his ability to derive phonology from words and pictures was severely impaired. He scored $6 / 20$ when required to point to a named nonword surrounded by other nonwords sharing letters but not pronunciations (e.g., vib, vit, vab). When asked to judge whether pairs of written words either rhymed or had the same pronunciation (i.e., with homophones), he again performed poorly. On the written-word rhyme-matching task from PALPA (Test 15), he scored 42/60 (70\% correct), which is significantly impaired in comparison with one age- and education-matched control (M.M., his wife; $58 / 60$ ) (Fisher exact probability, $p<.05$ ). On the homophone matching task from PALPA (Test 28), he scored
$34 / 60(57 \%)$ correct. His level of performance here did not differ from chance. The control, M.M., scored 57/60. J.M. scored 10/34 (29\%) in a test requiring him to point to two out of four pictures that had rhyming names, and in a rhyme-decision task requiring discrimination between two pictures that either had or did not have rhyming names, he scored 30/70 (43\%) correct. Given that J.M. was unable to access the correct phonology of words, we proposed that his ability to perform auditory wordwritten word and picture-matching tasks (see the Refractory Behavior section) was mediated by access to stored semantic knowledge for the items. On a number of tests, we showed that J.M. was able to access some semantic information about words and pictures, although his performance was usually outside the control range. For example, he scored 47/52 (90\%), 38/52 (73\%), and 44/52 $(85 \%)$ for pictures, written words, and auditory words, respectively, on the Pyramids and Palm Trees Test (Howard \& Patterson, 1992). These scores are below the normal control level (minimum 49/52 correct) for all modalities. On a picture-word matching test requiring discrimination between semantically related target and distractor items (from PALPA; Kay et al., 1992), J.M. scored 39/40 (98\%) with auditory word-picture matching and 31/40 (78\%) on written word-picture matching, which was significantly below his performance with auditory words (Fisher exact probability $=.007)$.

\section{Refractory Behavior}

The experiments in this section have already been reported in Forde and Humphreys (1995, 1997), but we provide here a brief summary of the procedures and key findings to demonstrate that J.M. showed all of the characteristic features of refractory behavior. We tested the effects of repetition and category on J.M.'s performance on auditory word-written word matching tasks. He was asked to match a spoken word to one of six categorically related written words. One trial was completed when all six words had been probed in a random order. The trial was then repeated using a different spatial layout of the written words and a different random order of presentation. A total of 28 categories were tested. The experiment was repeated on a separate occasion with six new words from each category. The key results are summarized in Table 1. J.M. was significantly better on the first (75\%) in comparison with the second $(66 \%)$ presentation $[\mathrm{McNemar}(1)=6.6, p<.05]$ that is, his performance became refractory as items were repeated. He was also better at matching some categories in comparison with others (see the Appendix). In particular, he was better at auditory word-written word matching for the names of famous people and places $(62 / 72,86 \%)$ than with common first names and surnames $(35 / 72,49 \%)\left[\chi^{2}(1)=\right.$ $23.03, p<.01]$. (For the same pattern in other "refractory" patients, see McNeil et al., 1994; Warrington \& McCarthy, 1987.) This experiment was repeated with pictures of the same items, and again J.M. was significantly better with the first $(123 / 132,93 \%)$ than with the second $(109 / 132,83 \%)$ presentation $[\mathrm{McNemar}(1)=7.6, p<.05]$.

We assessed the effect of rate on J.M.'s auditory wordpicture matching using the same paradigm with four cat- 
Table 1

J.M.'s Performance on Matching Tasks

\begin{tabular}{lcc}
\hline Effect of Repetition & P1 & P2 \\
\cline { 2 - 3 } Auditory word-written word matching & $252 / 336(75 \%)$ & $220 / 336(66 \%)$ \\
Auditory word-picture matching & $123 / 132(93 \%)$ & $109 / 132(83 \%)$ \\
Effect of Rate (Response-Stimulus Interval) & $2 \mathrm{sec}$ & $30 \mathrm{sec}$ \\
\cline { 2 - 3 } $\begin{array}{l}\text { Auditory word-picture matching } \\
\text { Effect of Semantic Distance }\end{array}$ & $54 / 72(75 \%)$ & $40 / 72(56 \%)$ \\
$\quad$ Auditory word-written word matching & Close & Distant \\
\cline { 2 - 3 } $\begin{array}{l}\text { Effect of Changing Input Modality } \\
\text { Same modality across four repetitions }\end{array}$ & P1 & P4/60 (90\%) \\
Change modality on fourth repetition & $53 / 60(88 \%)$ & $46 / 60(77 \%)$ \\
\hline Note- In all tests, there were six written words or pictures in an array, and J.M. was \\
asked to point to the one that matched an auditory word. Data taken from Forde and \\
Humphreys (1995, 1997).
\end{tabular}

egories (colors, fruits, vegetables, and geographical features) at a 2-sec and 30-sec response-stimulus interval. J.M. was significantly better with the slower rate of presentation $(54 / 72)$ than with the faster rate $(40 / 72)\left[\chi^{2}(1)=\right.$ $6.0, p<.02]$.

We assessed the effect of semantic distance on auditory word-written word matching with 12 categories. A trial consisted of each item in the array (of six words) being presented once in a random order. This was repeated three times consecutively for each array. J.M. was significantly worse in the close condition, when all six items were from the same category $(129 / 216,60 \%)$, than in the distant condition, in which they were all from different categories $(185 / 216,86 \%)\left[\chi^{2}(1)=36.6, p<.05\right]$.

In a further experiment, we evaluated whether the refractoriness could spread across modalities. J.M. was presented with an array of six exemplars from 1 of 10 categories. In one condition, he was given an auditory word-picture matching task, and the six items in the array were repeated four times consecutively. In another condition, he was given an auditory word-picture matching task on the first three presentations, but on the fourth presentation, the pictures were replaced by the corresponding words and the task changed to auditory word-written word matching. J.M. was significantly better on the first than on the fourth presentation in Condition $1\left[\chi^{2}(1)=\right.$ $3.84, p=.05]$, indicating that his performance had become refractory (see Table 1). Interestingly, he was also significantly worse on the fourth presentation in Condition 2 , even though the input of the stimuli had changed from pictures to written words $\left[\chi^{2}(1)=6.11, p=.01\right]$. We (Forde \& Humphreys, 1997) proposed that the most parsimonious explanation for this finding was to posit the locus of the refractoriness in a unitary semantic system (i.e., one that is accessed by pictures and words), rather than in modality-specific access procedures (though see Warrington \& Crutch, 2004, for a contrasting account).

\section{EXPERIMENT 1 Repetition Priming in Lexical Decision}

The Case Report illustrated that J.M.'s performance in picture-word and word--word matching tasks deteriorated when items were repeated, following the earlier probing of all of the items in the set. This contrasts with the more usual and very robust effect of repetition priming in normal subjects, which involves better performance on a task when stimuli are repeated than when they are presented for the first time (Jacoby \& Dallas, 1981; Salasoo et al., 1985). In the Case Report, we noted that J.M.'s ability to access the perceptual forms of words and pictures was relatively good, and, in the present experiment, we assessed whether J.M. could show repetition priming effects on a test of visual lexical decision. This test has been used to evaluate access to an orthographic input lexicon (Meyer \& Schvaneveldt, 1971), which, on at least some views, precedes access to semantic knowledge for written words (Coltheart, Rastle, Perry, Langdon, \& Ziegler, 2001). We were particularly interested to see whether J.M. could show repetition priming effects at the orthographic input lexicon for categories of items that consistently became refractory on tests that required access to semantic representations.

\section{Method}

J.M. was asked to perform a visual lexical decision task as quickly and accurately as possible. The stimuli were presented on an Apple Macintosh Quadra computer, which also measured J.M.'s reaction times (RTs). The stimuli were presented in 24-point Geneva font and remained on the screen until J.M. had responded. The response-stimulus interval was $500 \mathrm{msec}$. Since J.M. had use of only his left hand, he was asked to press the "C" key on a keyboard when the item was a word, and the " $\mathrm{X}$ " key when it was a nonword, with his fore and middle fingers, respectively. J.M. had used these two keys for binary response tasks in previous experiments and was comfortable with the procedure. We took 30 words from categories shown to be either impaired (girls' names, boys' names, surnames) or intact (countries, occupations, famous names) in J.M. in previous work (see Forde \& Humphreys, 1995, Appendix 1), and we derived 60 corresponding nonwords (e.g., germanw, plumfer, bacf) by changing one letter of the real words (germany, plumber, bach). On Trial 1, we presented J.M. with 30 words ( 15 from intact and 15 from impaired categories) and 30 nonwords in a lexical decision task. This task was repeated a second time, and then on Trial 3, we presented J.M. with all 60 words and 60 nonwords. We used an ABBA design, so on a separate occasion, the stimuli that had not been previously presented in Trial 1 and Trial 2 were tested in these trials, and the previously repeated items were presented for the first time on Trial 3. Critical comparisons were made between 
Table 2

Reaction Times (in Milliseconds) for Words in Visual Lexical Decision Task (Experiment 1)

\begin{tabular}{|c|c|c|c|c|c|c|}
\hline \multirow[b]{2}{*}{ Category } & \multicolumn{2}{|c|}{$\mathrm{T} 1$} & \multicolumn{2}{|c|}{$\begin{array}{c}\mathrm{T} 3 \\
\text { (Repeated) } \\
\end{array}$} & \multicolumn{2}{|c|}{$\begin{array}{c}\text { T3 } \\
\text { (New) }\end{array}$} \\
\hline & $M$ & Err & $M$ & Err & $M$ & Err \\
\hline Impaired & 2,559 & 9 & 2,870 & 5 & 4,085 & 13 \\
\hline Intact & 3,715 & 8 & 2,988 & 8 & 4,432 & 7 \\
\hline
\end{tabular}

performance on Trial 1 and performance on Trial 3 with repeated $[\mathrm{T} 3(\mathrm{R})]$ and nonrepeated $[\mathrm{T} 3(\mathrm{~N})]$ items.

\section{Results and Discussion}

J.M.'s mean correct RTs and accuracy data for Trials 1 and 3 are shown in Table 2. In terms of overall accuracy, J.M. was above chance, although far from perfect. He correctly classified $130 / 180(72 \%)$ of the words and $125 / 180$ $(69 \%)$ of the nonwords. Seven RT outliers $(>2.5 S D)$ were removed from the analysis, and the remaining data formed a normal distribution. We performed a two-way ANOVA on J.M.'s correct RTs for words, treating each RT as a separate subject. There was a significant main effect of condition $($ Trial 1 ; Trial 3 repeated vs. Trial 3 new) $[F(2,117)=$ $6.02, p=.003]$ but not of category (impaired vs. intact) $[F(1,117)=2.626, p=.11]$, and no significant interaction $(F<1) .{ }^{2}$ A Student Newman-Keuls test showed that there was a significant difference between items that were presented new on the third trial $(4,281 \mathrm{msec})$ in comparison with items that were repeated on the third trial $(2,924 \mathrm{msec})$ and in comparison with Trial $1(3,166 \mathrm{msec})$. The magnitude of the priming effect between $\mathrm{T} 3(\mathrm{~N})$ and $\mathrm{T} 3(\mathrm{R})$ was $1,444 \mathrm{msec}[32 \%$ of $\mathrm{T} 3(\mathrm{~N})]$ for intact categories and $1,215 \mathrm{msec}[30 \%$ of $\mathrm{T} 3(\mathrm{~N})]$ for impaired items.

Interestingly, there was no significant difference in J.M.'s lexical decision times, or accuracy, for categories that had previously been intact $(3,734 \mathrm{msec})$ or impaired $(3,116 \mathrm{msec})$ in auditory word-written word matching (Forde \& Humphreys, 1995). J.M. was, however, significantly faster on lexical decision for items that were repeated (Trial 3 repeated) than for those that were presented for the first time on the final trial (Trial 3 new). There was no overall RT advantage for items repeated on Trial 3 [T3(R)] relative to when the same items were presented on Trial 1. However, this contrast is confounded by possible effects of fatigue, and we propose that the contrast between repeated and nonrepeated items on Trial $3[\mathrm{~T} 3(\mathrm{R})-\mathrm{T} 3(\mathrm{~N})]$ provides a purer assessment of repetition effects in patients with brain damage.

The contrast between the benefit from repetition on lexical decision and the generally negative effects of repetition in other tasks (see above) can be conceptualized in various ways. One account attributes the contrast to effects occurring at different levels of the language processing system. Lexical decision may reflect activation in an orthographic lexicon, and prolonged activation in the lexicon may benefit responses to the same word when it is re-presented. In contrast, tasks in which J.M. typically performed poorly (e.g., picture-word matching) may require access to semantic knowledge. Prolonged activation and/or abnormal inhi- bition at a semantic level, however, may make it difficult to reaccess an individual response to a stimulus - especially if activation spreads to the representations of related items, which then compete for selection for a response. Alternatively, lexical decisions could be facilitated here because there is priming of a learned motor response to a given stimulus (e.g., "press this button to this particular item"). In Experiment 2, we examined the relations among repetition priming, lexical decision, and picture-word matching more directly. Items were initially repeated in a picture-word matching task, in which J.M. previously showed refractory behavior, and then we immediately re-presented the stimuli for lexical decision. Thus, we directly tested whether items that entered a refractory state in the first task could nevertheless benefit from that earlier presentation when the subject performed the second task. Note, also, that any benefit from repetition on lexical decision here cannot be attributed to priming at a motor level, since different responses were demanded on the first presentation (picture-word matching) and the repeated presentation (lexical decision).

\section{EXPERIMENT 2 Refractoriness and Repetition Priming}

\section{Method}

In Part 1, J.M. was presented with arrays of six words from a particular category. He received three arrays with exemplars from intact categories (countries, occupations, famous names) and three arrays with exemplars from impaired categories (surnames, boys' names, girls' names). The experimenter (E.M.E.F.) presented the name of one of the stimuli auditorily, and J.M. was asked to point to the corresponding written word. When all six exemplars had been probed, the stimuli were rearranged and the procedure was repeated an additional three times to give a score correct out of 24 for each array. This was repeated for all six categories. In Part 2, which followed immediately after the last trial in Part 1, J.M. was given a visual lexical decision task and was asked to respond as quickly and accurately as possible. The procedure for this was the same as in Experiment 1. There were 144 stimuli randomly presented (72 words and 72 nonwords). Half of the words were the same ones that had been used in Part 1, and the other half were six new exemplars from the same categories. Nonwords were created by changing one letter in each of the words.

On a separate occasion, the experiment was repeated to counterbalance the stimuli. Items that had previously been presented for auditory word-written word matching in Part 1 became the new exemplars in the lexical decision task. Items that had only been presented in the lexical decision task on the first occasion were now used as stimuli for auditory word-written word matching, and were repeated for the lexical decision task.

\section{Results and Discussion}

The mean correct RTs and errors are shown in Table 3. In the auditory word-written word matching task, J.M. became significantly worse on Trial 4 (11/36) in comparison with Trial $1(23 / 36)$ for the impaired categories $\left[\chi^{2}(1)=\right.$ $8.0, p<.01]$. There was no significant effect of repeating the auditory word-written word task for the intact categories, 35/36 for Trial 1 and 34/36 for Trial 4. This replicates previous findings that showed that J.M. was significantly better with some categories (countries, famous names, occupations) than with others (surnames, boys' names, girls' names), and that for impaired categories, his performance 
Table 3

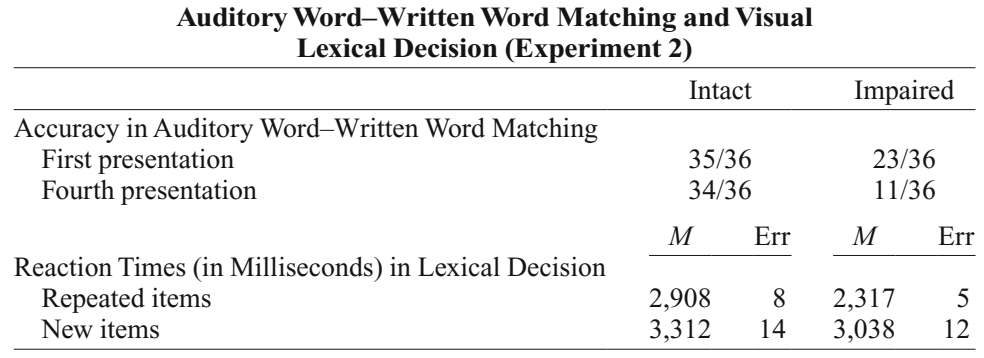

became refractory over repetitions (Forde \& Humphreys, 1995, 1997).

In the lexical decision task, J.M.'s overall performance in terms of accuracy was again well above chance, although not perfect. He correctly classified 100/139 (72\%) of words and 113/141 (80\%) of nonwords. For words, he was significantly better on repeated items $(81 \%)$ than on new items $(63 \%)\left[\chi^{2}(1)=5.9, p=.02\right]$. There was no difference in his accuracy between intact $(75 \%)$ and impaired $(69 \%)$ items $\left[\chi^{2}(1)<1.0\right]$. For the RT data in the lexical decision task, we removed five machine errors and four RT outliers $(>2.5 S D)$ to generate a normal distribution. A two-way ANOVA was performed on J.M.'s RTs for items correctly classified as words. His RTs showed the same pattern as the accuracy data, and again J.M. benefited from the repetition of items, although the difference between repeated $(2,601 \mathrm{msec})$ and new $(3,163 \mathrm{msec})$ items just failed to reach statistical significance $[F(1,92)=$ $2.95, p=.09]$. There was no effect of category (impaired, intact) $[F(1,92)=1.74, p=.19]$ and no interaction between category and condition $[F(1,92)=0.24, p=.63]$. J.M. showed a $723-\mathrm{msec}$ priming effect for impaired items (i.e., items that became refractory on the matching task) and a 404-msec priming effect for the intact items.

These results indicate that items from impaired categories can become refractory (in a behavioral sense) in auditory word-written word matching tasks, and yet the same items still showed facilitatory repetition priming effects in lexical decision when they were presented immediately after the auditory word-written word matching task. The facilitatory effect of repetition on lexical decision may be explained by proposing that the lexical representations of repeated items are more highly activated than those of new items (Schacter \& Cooper, 1993), or it might be attributed to changes in the weights connecting letters to their lexical representations (cf. Monsell, 1990). In either case, such effects may be separated from effects operating at other levels of the language system (e.g., within the semantic system), where J.M.'s impairment might lie. On this view, J.M.'s semantic representations may become inhibitory on repeated testing, and they cannot be accessed to support picture-word matching (McCarthy \& Kartsounis, 2000). However, providing that lexical decisions can be based on stored lexical but nonsemantic knowledge (Coltheart, 1978), facilitatory effects of lexical preactivation, or of stimulus-specific weight changes, can still be observed, even if semantic information about the same items is inaccessible. Consistent with this view that lexical decisions were based on nonsemantic information is that there was no effect of the category of item on J.M.'s lexical decision performance, though item category strongly affected picture-word matching. One other view is that J.M.'s refractory behavior arises because there is some degree of semantic impairment coupled with abnormal persistence of activation within the semantic system. In the Case Report, we noted evidence that J.M. had some degree of semantic impairment (e.g., a mild deficit on Pyramids and Palm Trees; Howard \& Patterson, 1992). In this case, there may well be partial activation of semantic competitors when an item is first presented. Following the presentation of the remaining competitors from the same semantic set, there would even be a spread of activation within the target's field, making selection particularly difficult if targets have to be discriminated from semantic competitors for task performance (as with picture-word matching; see Forde \& Humphreys, 1995). This account too would fit with the idea that facilitatory effects of repetition emerge on lexical decision, because that task taps presemantic representations. However, it could also be that lexical decisions are mediated by access to semantic knowledge (cf. Plaut, McClelland, Seidenberg, \& Patterson, 1996), but that persistent activation at a semantic level then benefits rather than impairs performance. The crucial difference between lexical decision and picture-word matching can be attributed to the specificity of the semantic information that needs to be accessed. Picture--word matching depends on access to finegrained semantic information, which deteriorates under conditions in which fine-grained knowledge is degraded and there is increased competition between neighbors (after repeated presentation of the stimuli). In contrast, lexical decisions may be made even if there is activation across a set of semantic neighbors, since this activation would be consistent with the stimulus being a known word.

Other accounts of the relations between picture-word matching and lexical decision here seem less viable. For example, one possibility is that both tasks are dependent on access to semantic knowledge, but that activation in J.M.'s semantic system shows a U-shaped function; after initial access to semantic information, the representations involved are first inhibited (over the three repeats used here) and then activated above baseline (to produce facilitation in lexical decision). However, we know of no converging evidence in the literature that would match such a U-shaped function for semantic activation - such a pattern would need to have resulted from J.M.'s brain lesion, which, though possible, 
seems unlikely. Nevertheless, in order to provide more direct tests of these different accounts, we conducted Experiments 3 and 4 .

\section{EXPERIMENTS 3 AND 4 Semantic Priming}

In these experiments, we examined whether repetition led to prolonged activation and competition within J.M.'s semantic system, or whether it led to inhibition of his semantic representations, and we tested whether the pattern of data in Experiment 2 was likely to have been caused by just the temporal parameters of testing. The first task (Experiment 3) was picture-word matching, which required J.M. to match detailed semantic information accessed from both the word and the picture to succeed, since he could not access phonological information about words or pictures to match at a phonological level (see the Case Report). In the second task (Experiment 4), we asked him to make a living/nonliving judgment, which required access to only general (common) features of objects. In Forde and Humphreys $(1995,1997)$, we showed that J.M. could access superordinate semantic knowledge about stimuli even when he failed to access a precise semantic representation (e.g., when asked to point to written names, he made errors by pointing to the wrong name that was nevertheless corresponding to the same sex as the target's name). The important point is that in both experiments, J.M. was required to access stored semantic representations, and the temporal relations between primes and targets were similar in both studies. However, the studies differed in the level of information required to successfully complete the task. If there is inhibition of semantic knowledge with the temporal parameters employed in Experiment 3 (requiring access to detailed semantic information), then this should also be observed in Experiment 4 (requiring access to superordinate semantic knowledge) — on the basis of the inhibitory and time-based accounts of the previous studies. On the other hand, if there is prolonged activation of degraded semantic knowledge, then competition between semantic neighbors may lead to refractory behavior when fine-grained knowledge has to be accessed (Experiment 3), but facilitated performance when superordinate knowledge alone is required (Experiment 4).

\section{EXPERIMENT 3 Picture-Word Matching}

In this experiment, we modified a paradigm used by Blaxton and Bookheimer (1993), who showed that a group of anomic subjects were slower to read target words that followed semantically related primes than they were to read target words that followed unrelated primes. Blaxton and Bookheimer suggested that this was due to a form of category-specific inhibition of name retrieval. Previous work on J.M. and other access-dysphasic patients in the literature has shown a decline in performance when the target is presented simultaneously with other categorically related words (Forde \& Humphreys, 1995, 1997; Warrington \& McCarthy, 1983, 1987), and here we test whether refractoriness can also occur when related items are presented sequentially. To investigate the influence of semantic primes on access to fine-grained semantic information, we used a picture-word matching task.

\section{Method}

A word and a picture were presented simultaneously on the screen of an Apple Macintosh Quadra computer, and J.M. was asked to decide as quickly and accurately as possible whether the stimuli matched. In all trials, there were three picture-word primes and one picture-word target. J.M. made a two-choice keypress response with the fore and middle fingers of his left hand to indicate whether the stimuli matched or not. All of the pictures were line drawings taken from the Snodgrass and Vanderwart (1980) set. Nonmatching trials were created by swapping words within a category so they no longer corresponded to the name of the picture. Responses (match or no match) varied for both primes and targets, and primes could be either from the same category as the target or not. If the primes matched, there was an equal chance of the target belonging to the same (e.g., dog-dog, cat-cat, horse-horse, cow-cow) or a different category as the primes (e.g., hammer-hammer, axe-axe, nail-nail, cow-cow), and there was an equal chance that items from the same category matched (e.g., dog-dog, cat-cat, horse-horse, cow-cow) or did not match (e.g., dog-dog, cat-cat, horse-horse, cow-sheep). If the primes did not match, there was an equal chance that targets matched (e.g., dog-cat, horse-dog, cat-horse, cow-cow) or did not match (e.g., dog-cat, horse-dog, cat-horse, cow-sheep).

These conditions were chosen because J.M. then had the same likelihood of making either the same or a different response to the target as to the primes in the same- and different-category conditions, so that category similarity was irrelevant to the task. In order to provide a "neutral" baseline, uncontaminated by any effect of the preceding primes, we also ran a block of trials in which J.M. made picture-word matching responses to targets presented alone. An ageand background-matched control (M.M.) performed the experiment under the same conditions.

\section{Results and Discussion}

For J.M., we applied a one-way ANOVA to the correct RTs for matching trials for the following conditions: target alone (carrot-carrot), target preceded by three matching primes from the same category (celery-celery, mushroommushroom, potato-potato, carrot-carrot), and target preceded by three matching primes from a different category (cup-cup, glass-glass, kettle-kettle, carrot-carrot). The data from trials with nonmatching items were not analyzed, because it is unclear what information would be recruited in order to decide that the items differed, and nonmatching responses might be affected by postsemantic access check processes conducted by participants (much as "no" responses in present-absent search tasks are difficult to interpret because they can be dependent on postsearch check processes; see Chun \& Wolfe, 1996). There was a significant main effect of condition on matching trials $[F(2,49)=$ $3.8, p=.03]$. A post hoc test (Student Newman-Keuls) showed that J.M. was significantly slower when the target was preceded by categorically related primes $(3,288 \mathrm{msec})$ than in the target-alone condition $(2,266 \mathrm{msec})$ and the condition in which the target had been preceded by unrelated primes $(2,396 \mathrm{msec})$. The magnitude of the detriment for 
Table 4

Reaction Times (in Milliseconds) for Matching Pairs in Picture-Word Matching (Experiment 3)

\begin{tabular}{|c|c|c|c|c|c|}
\hline \multirow[b]{2}{*}{ Condition } & \multirow[b]{2}{*}{ Example } & \multicolumn{2}{|c|}{ J.M. } & \multicolumn{2}{|c|}{ M.M. } \\
\hline & & $M$ & Err & $M$ & Err \\
\hline Target alone & cow-cow & 2,266 & 1 & 643 & 2 \\
\hline 3 same $(3 \mathrm{~S})$ & dog-dog, cat-cat, horse-horse, cow-cow & 3,288 & 2 & 612 & \\
\hline 3 different (3D) & hammer-hammer, axe-axe, nail-nail, cow-cow & 2,396 & 5 & 690 & \\
\hline
\end{tabular}

related relative to unrelated primes was $892 \mathrm{msec}$. These results are summarized in Table 4. There was no evidence of a speed-accuracy trade-off.

We also applied a one-way ANOVA to the correct RTs of the control subject for the same conditions. The control subject showed a strong trend in the opposite direction to that for J.M. $[F(2,55)=2.72, p=.07]$. She was faster with targets preceded by three primes from the same category $(612 \mathrm{msec})$ than with the target alone $(643 \mathrm{msec})$ or with the target preceded by three primes from a different category $(690 \mathrm{msec})$.

The results showed that J.M. became significantly slower at deciding that a written word was the name of a picture when the stimuli were preceded by word-picture pairs from the same category in comparison with both an unprimed condition and a condition in which word-picture pairs came from a different category. In other words, his performance became refractory when he had to access finegrained semantic information about particular items. This result stands in clear contradiction to the data on lexical decision, which showed that J.M. was speeded by repetition (Experiments 1 and 2). The result suggests that there can be opposite effects of repetition on performance, depending on the level in the language system being "tapped" by the particular task or on the specificity of the semantic information that must be retrieved. However, it is also the case that targets were probed here over a shorter interval than in the critical lexical decision probes in Experiment 2 (which followed after the block of picture-word matching trials). In the next experiment, we assess whether J.M.'s semantic system necessarily becomes refractory when superordinate semantic knowledge is probed, keeping the time parameters close to those used in Experiment 3.

\section{EXPERIMENT 4A Superordinate Categorization}

\section{Method}

J.M. was asked to categorize words into living or nonliving categories as quickly and accurately as possible, using two designated letters on a keyboard - " $\mathrm{X}$ " for living and " $\mathrm{C}$ " for nonliving. The words were presented on, and RTs measured by, an Apple Macintosh Quadra computer. The words were again presented in 24-point Geneva font; they remained on the screen until J.M. responded. A fixation cross (presented for $500 \mathrm{msec}$ ) marked the start of a new trial, which could consist of target alone, T (e.g., apple), or target proceeded by: three primes from the same category, $3 \mathrm{~S}$ (e.g., $b a-$ nana, cherry, grapes, apple); one prime from the same category, $1 \mathrm{~S}$ (e.g., banana, apple); three primes from another category, 3D (e.g., chisel, spanner, screw, apple); or one prime from another category, 1D (e.g., chisel, apple). There were 20 target words (10 living and 10 nonliving), which appeared in all five conditions, presented in a random order. Items were taken from a number of categories: animals, birds, fruits, and vegetables for the living categories; tools, furniture, clothes, and kitchen items for the nonliving categories. All of the items were imageable and predominantly in the intermediate range of J.M.'s performance in previous pictureword matching experiments (Forde \& Humphreys, 1995). Our control subject for J.M. (M.M.) performed the experiment under the same conditions.

\section{Results}

The mean correct RTs and accuracy data for J.M. are shown in Table 5. We removed seven machine errors and one RT outlier $(>2.5 S D)$, and performed a one-way ANOVA on the correct RTs. There was a significant main effect of condition $[F(4,85)=3.64, p=.009]$. A Student NewmanKeuls test $(p=.05)$ demonstrated that J.M. was significantly faster when the target had been preceded by three primes from the same category $(966 \mathrm{msec})$ in comparison with the baseline condition of target alone $(1,413 \mathrm{msec})$ and in comparison with when it had been preceded by one prime from the same category $(1,348 \mathrm{msec})$. There was no significant difference between the target-alone condition and the one unrelated prime $(1,134 \mathrm{msec})$ or the three unrelated prime $(1,339 \mathrm{msec})$ conditions. The RT difference between the $3 \mathrm{~S}$ condition and the target-alone condition was $447 \mathrm{msec}$ ( $32 \%$ of target-alone RT). J.M. made only two errors, one in the $3 \mathrm{~S}$ and one in the $1 \mathrm{D}$ condition.

We removed one machine error and applied a one-way ANOVA to the correct RTs of the control subject, M.M. There was a significant effect of condition $[F(4,88)=$ $10.01, p<.001]$. A post hoc test showed that she was sig-

Table 5 Reaction Times (in Milliseconds) for Superordinate Categorization (Experiment 4)

\begin{tabular}{|c|c|c|c|c|}
\hline \multirow[b]{2}{*}{ Condition } & \multicolumn{2}{|c|}{ J.M. } & \multicolumn{2}{|c|}{ M.M. } \\
\hline & $M$ & Err & $M$ & Err \\
\hline \multicolumn{5}{|c|}{ Experiment 4A } \\
\hline 3 same category (3S) & 966 & 1 & 560 & \\
\hline 1 same category (1S) & 1,348 & & 598 & \\
\hline Target alone $(\mathrm{T})$ & 1,413 & & 715 & \\
\hline 1 different category (1D) & 1,134 & 1 & 719 & 3 \\
\hline 3 different category (3D) & 1,339 & & 737 & 3 \\
\hline \multicolumn{5}{|c|}{ Experiment 4B } \\
\hline 3 same category $(3 S)$ & 906 & & 655 & \\
\hline 3 same category and 1 repeat (3SR) & 892 & & 651 & \\
\hline Target alone $(\mathrm{T})$ & 1,210 & 2 & 799 & 2 \\
\hline 3 different category and 1 repeat (3DR) & 1,275 & 2 & 728 & \\
\hline 3 different category (3D) & 1,112 & & 732 & \\
\hline
\end{tabular}


nificantly faster when the target was preceded by three categorically related primes $(560 \mathrm{msec})$ and by one categorically related prime $(598 \mathrm{msec})$ in comparison with each of the target-alone $(715 \mathrm{msec})$, the three unrelated primes $(719 \mathrm{msec})$, and the one unrelated prime $(737 \mathrm{msec})$ conditions. The RT difference between the $3 \mathrm{~S}$ and the target-alone condition was $155 \mathrm{msec}$ ( $22 \%$ of target-alone RT). M.M. made six errors, three in the $1 \mathrm{D}$ and three in the $3 \mathrm{D}$ condition.

\section{Discussion}

J.M. showed a facilitatory semantic priming effect when targets were preceded by categorically related items, particularly after three related primes had been presented. This contrasts with his performance on picture--word matching (Experiment 3), when pairing a target with related items impaired his performance. It is of interest to note that J.M. showed a larger priming effect relative to the control subject (benefits of $32 \%$ vs. $22 \%$, in comparison with the targetalone baseline condition, for J.M. and M.M., respectively). In studies of semantic priming effects in patients with Alzheimer's disease, Chertkow, Bub, and Seidenberg (1989) also reported relatively large, "hyperpriming" effects, and argued that such hyperpriming effects could be considered characteristic of an impaired semantic memory system. This may occur because patients lose the fine-grained semantic information necessary to distinguish between items; in this case, semantic priming may become a form of repetition priming. Our Case Report illustrates that J.M. had some loss of fine-grained semantic information too, so any enhanced semantic priming may reflect this. Nevertheless, the important result is that semantic priming here facilitated performance, and there was no evidence for inhibition at the superordinate level. It is also important to note that the result occurred even though the timing relations between primes and target were similar to those in Experiment 3 (when refractory effects were observed). This runs counter to the argument that timing relations between primes and targets were critically important for the differences between refractory and facilitatory effects of priming (e.g., in Experiment 2).

In Experiment 4B, we tested the effects of semantic priming using a modified version of this paradigm, in which targets were repeated within a stimulus string. The effects of repetition were examined on items presented within a context of stimuli from either the same or a different category.

\section{EXPERIMENT 4B Superordinate Categorization and Repetition}

\section{Method}

The stimulus presentation conditions matched those used in Experiment $4 \mathrm{~A}$, and the same words were used as primes and targets. J.M. was asked to categorize words into living or nonliving categories as quickly and accurately as possible, and his RTs were measured. A fixation cross (presented for $500 \mathrm{msec}$ ) marked the start of a trial, which consisted of a target (e.g., apple) preceded by either: three primes from the same category (3S: banana, cherry, grapes, apple); the target and two primes from the same category (3S repeated: apple, banana, cherry, apple); three primes from a different category (3D: chisel, spanner, screw, apple); or the target and two primes from a different category (3D repeated: apple, chisel, spanner, apple). RTs for the first item in the $3 \mathrm{~S}$ repeated and $3 \mathrm{D}$ repeated conditions were taken as a baseline for a "no-priming" condition. The control subject (M.M.) performed the experiment under the same conditions.

\section{Results}

We removed three RT outliers $(>2.5 S D)$ and performed a one-way ANOVA on J.M.'s correct RTs. There was a significant main effect of condition $[F(4,108)=2.85, p=$ .03], and as can be seen from Table 5, J.M. was better when targets were preceded by semantically related primes than he was in the target-alone or the unrelated prime conditions. However, there was no clear effect of repeating an item within a trial. To examine the effects of repetition and relatedness in more detail, we applied a two-way ANOVA to the nested combinations of data for the repetition and relatedness conditions. J.M. was significantly faster with related $(899 \mathrm{msec})$ than with unrelated $(1,189 \mathrm{msec})$ items $[F(1,71)=11.75, p=.001]$, but there was no main effect of repetition $[F(1,71)=1.07, p=.31]$ and no significant interaction $[F(1,71)<1]$. The magnitude of the difference between the related and unrelated conditions was $290 \mathrm{msec}$ ( $25 \%$ of the unrelated condition baseline). J.M. was generally accurate, and there was no evidence of a speedaccuracy trade-off.

We also applied a one-way ANOVA to the correct RTs of the control subject, M.M., and found a significant effect of condition $[F(4,108)=7.71, p=.0001]$. Her RTs are given in Table 3 . We examined the effects of repetition and relatedness using an ANOVA analogous to that conducted with J.M. Like J.M., M.M. was significantly faster with related items $(653 \mathrm{msec})$ than with unrelated items $(730 \mathrm{msec})[F(1,75)=8.77, p=.004]$, but there was no effect of repetition $(F<1)$ and no interaction between the variables $(F<1)$. The magnitude of the facilitation effect between related and unrelated items was $77 \mathrm{msec}(11 \%$ of the unrelated baseline condition).

\section{Discussion}

Experiment 4B replicated the facilitatory priming effect of preceding a target with categorically related items that was found in Experiment 4A. In addition, there was no significant difference between the $3 \mathrm{~S}$ and $3 \mathrm{~S}$ repeated, or between the $3 \mathrm{D}$ and $3 \mathrm{D}$ repeated, conditions; repetition of an item did not produce a decline in performance. The present facilitatory effect between semantically related items contrasts with the data from Experiment 3, in which J.M. showed refractory behavior on accessing semantic information when targets were preceded by related primes. It seems that it is only when J.M. is asked to perform a task that requires access to fine-grained semantic information (e.g., picture-word matching) that his performance is hampered by the presence of semantically related items - either when these items are presented simultaneously with the target (Experiment 2) or preceding the target (Experiment 3 ). Access to superordinate semantic information, however, is facilitated by preceding the target with items from the same category. The most straightforward account of this is that there is a spread of activation between neighbors of individual items at the semantic level, with this leading to stronger activation of superordinate semantic representa- 
tions. Alternatively, the result could be consistent with a refractory account if representations of individual items are suppressed, so there is less differentiation between items within categories. There may then be more efficient assignment of items to their superordinate category.

In contrast to the beneficial effect of having items from the same categories, there was no effect of repetition, for either J.M. or the control, M.M. This might reflect the fact that access was only required to superordinate information, in which case activation of the precise semantic representation for one item had no differential impact relative to there being activation of a number of semantically related neighbors. Regardless of this, there was no evidence here for inhibitory effects at a semantic level.

An alternative account of the facilitation effects in Experiment 4 is that J.M. was sensitive to priming at a motor response level. In the same category conditions, primes and target belonged not only to the same semantic category but also to the same response class. Priming of the response could have facilitated performance. However, although this proposal may be applied to Experiment 4, the same should also hold for some of the critical conditions in Experiment 3, when a matching response to a target was preceded by matching responses to primes from the same category (e.g., in the $3 \mathrm{~S}$ condition, in comparison with when the target was presented alone). Yet in Experiment 3, J.M. was disrupted by having related primes (relative to the target along baseline). To explain the data from Experiment 3, we need to suppose that any response priming effect was overruled by an inhibitory effect from related items. The question is why this was not the case in Experiment 4 too, when primes and targets were related. We suggest that this reflects the specificity of representations that need to be accessed at a semantic level. There is refractory behavior only when specific representations must be accessed. We propose that this can be accounted for in terms of J.M. having difficulty in making fine-grained semantic distinctions, either when multiple semantic representations are activated (an account in terms of activation and competition) or when item-specific semantic representations are suppressed (a refractory account). A motor priming explanation also fails to explain all aspects of J.M.'s performance in Experiment 4. If there was motor priming, we would expect a large "switch effect," when a response changed from "living" to "nonliving." This was not the case. There was no significant difference between J.M.'s performance when a target was preceded by three primes that required a different response to the target $(1,339 \mathrm{msec}$, Condition 3D) relative to the target-alone conditions $(1,413 \mathrm{msec})$. We conclude that motor response priming was not critical here.

The data do support our prior work with J.M. though. In Forde and Humphreys (1997, Experiment 3) we reported that J.M. did not show refractory behavior when items were repeated at a superordinate level. In that study, J.M. received two superordinate labels and six names (three for one category and three for the other). J.M. had to point to the superordinate label for each item. Even though items were probed in a random order (so that consecutive trials went across categories as often as within categories), J.M. did not become worse over trials. In contrast, in formally equivalent picture-word matching tasks requiring access to the semantic representations of specific items, J.M.'s accuracy at matching did decrease with repetition. Again, there is a contrast between performance when specific and superordinate semantic information had to be retrieved; the deficit was found only when specific information had to be accessed.

\section{GENERAL DISCUSSION}

In this article, we have investigated the effect of repetition and semantic priming in a patient, J.M., previously shown to have the defining features of a category-specific access dysphasic (Forde \& Humphreys, 1995, 1997). In Experiment 1 , we examined the effect of repetition in a lexical decision task and found that J.M. benefited from prior exposure of the target. This contrasts with previous work, which found that J.M.'s performance declined, or became refractory, as an item was repeated (see Forde \& Humphreys, 1995, 1997, and the Case Report above). In Experiment 2, we examined the relationship between refractoriness and repetition priming in more detail. We found that, using the same items, words that had become refractory on tasks requiring auditory word-written word matching were nevertheless responded to more accurately and more quickly than new items, when presented immediately afterward in a lexical decision task. In Experiments 3 and 4, we assessed whether J.M. would benefit from priming by semantically related items. We found that, in pictureword matching, J.M.'s performance declined when the target was preceded by semantically related primes in comparison with unrelated primes and with targets presented alone (i.e., his performance became refractory over time). However, when items were presented over the same time interval but the task was changed to superordinate categorization (in Experiment 4), he showed facilitatory priming (in both Experiments 4A and 4B). Furthermore, in contrast to both previous work (Forde \& Humphreys, 1995, 1997) and to the auditory word-written word matching task in Experiment 2, repeating items within a trial did not affect his performance.

A number of accounts of the data can be ruled out. One account is that J.M. shows facilitatory effects of response/ motor priming, but disruptive effects of repetition/semantic relatedness on access to stored knowledge representations. Although a motor priming account can explain the benefit for repeated lexical decisions in Experiment 1, it fails to explain the positive effects on lexical decision from previously having made a picture-word matching response (Experiment 2). Also, although response repetition could have contributed to the data when superordinate responses were made (Experiment 4), it should also have contributed positively to performance in Experiment 3, since the same (matching) response was then made across the critical items. Response priming alone fails to account for the contrasting patterns of performance. A further suggestion is that the refractory and facilitatory priming effects arise 
at different time intervals. In Experiment 2, for instance, immediately repeated picture-word matching tasks were impaired, whereas subsequent lexical decision responses were facilitated. It could be that this later facilitation effect reflected a distinct process of long-term learning (e.g., retuning weights between representations), or a U-shaped activation function (inhibition followed by activation), even if the representation itself was initially refractory. However, this fails to explain the contrast between Experiments 3 and 4 (refractory behavior in Experiment 3, facilitation effects in Experiment 4), when the timing between the events was very similar.

We consider below other accounts of the results and their implications for understanding semantic processing.

\section{What Is Refractoriness?}

The concept of refractoriness has been developed to account for the pattern of performance shown by patients with category-specific access dysphasia (Warrington \& McCarthy, 1983, 1987). The term describes a pattern in which performance declines when target stimuli are presented repeatedly. One account of this is that the representation of a target item (or the access procedures to that representation) becomes temporarily inhibited following its activation (see McCarthy \& Kartsounis, 2000). A spread of this refractory state between items in the same neighborhood, or to items sharing elements of the same representation, leads to the problem being most pronounced when items are semantically related. An alternative is that refractoriness in behavior may occur because the activation of target items, and of their semantically related neighbors, remains abnormally high following stimulus presentation. This, in turn, makes it more difficult to select the target from other highly activated representations, when items are returned to after all of them have been probed once (the typical procedure in studies of refractory behavior in patients). The difficulty will be particularly severe if there is a semantic impairment so that it is already difficult to discriminate between items with overlapping semantic features, and it will affect performance particularly when precise semantic information needs to be accessed for the task. This is the case for J.M., who performed below normal levels on tests requiring access to semantic memory, even when items were only presented once (see Case Report).

The evidence presented here is consistent with there being prolonged activation of representations, so that, for example, having carried out picture-word matching in an earlier part of a study would facilitate lexical decisions to the same items (relative to when items appear for the first time). ${ }^{3}$ Similarly, activation of one item from the same category then facilitates superordinate decision making to an item that follows it belonging to the same category. In Experiment 2, we showed that the "refractory" items, far from being inhibited, appeared to remain activated, and produced facilitatory priming on a subsequent lexical decision task. Experiment 4 provided converging evidence that facilitation was not confined to decisions made at a lexical level, because superordinate responses were also facilitated by semantic priming. The most parsimonious explanation of these last results is that the semantic representations of stimuli remained activated for an abnormally long period following target selection. Due to this prolonged activation, several representations are available, making difficult the selection of fine-grained semantic information for a target.

Although these results are consistent with the idea that "refractory patients" in fact suffer abnormal activation and competition, the data can still be explained in terms of a refractory account, if there are different effects at different levels of representation in the language system. For example, at the level of individual item semantics, J.M. may suffer from refractory representations, which cannot be activated for some period after first being probed. At a lexical level, though, there may be either continued lexical activation or a change of connectivity within the lexicon so that there is faster processing (and there are faster lexical decisions) to repeated items (Experiment 2). Similarly, superordinate nodes in the semantic system may remain activated even if individual semantic representations are refractory, so there is facilitatory priming of category decisions between items from the same category (Experiment 4 ). A further possibility is that the refractory state of semantics for individual items helps superordinate classification by making individual items within a category more similar, due to individuating semantic features being in a refractory state. Although these accounts are less parsimonious than the activation and competition account, they remain viable. Indeed, using similar presentation intervals as were used in the conditions in which facilitatory semantic priming occurred in Experiment 4, we found interference effects from semantic priming in Experiment 3 when the task required access to detailed semantic information (for picture-word matching). This last result suggests that the specificity of the semantic information required was critical for task performance.

One possible way of testing the theories that refractoriness reflects abnormal levels of inhibition or activation might be to assess whether patients show perseverative or even "reverse perseverative" behavior in tasks such as auditory word-written word and picture matching. The "inhibition" account would predict that once an item had been activated, it would be abnormally inhibited, and consequently patients would be less likely to select it on a subsequent trial. That is, there should be a form of reverse perseveration, in which a previously probed target would be less likely to be responded to (when another item is prompted) than items previously unprobed. The "activation" account predicts that patients would show neither perseverative nor reverse perseverative effects. At first sight, this prediction is counterintuitive. One might expect J.M. to perseverate if items remain abnormally activated, since activated representations could reach their access thresholds earlier than competitors. However, in previous work, we have demonstrated that the refractory behavior spreads within a category (Forde \& Humphreys, 1995), so that there are (apparently) many representations rather than a single semantic representation active (particularly if semantic representations are distributed rather than localized). Hence, there is little reason to think that J.M. will show perseverative (or reverse perseverative) responses to any one particular item. To test 
these predictions, we reanalyzed data from two experiments reported previously in Forde and Humphreys (1995). In the first data set (Forde \& Humphreys, 1995, Experiment 6, Set 2), we had recorded order data for items in a trial and were able to assess whether FK's erroneous responses were perseverations of previously presented items. We found that he was as likely to select a response before it was presented (18/37 errors) as he was to perseverate with a previously presented response (19/37). There was neither an increase nor a decrease in responding to an item after it was probed. In the second reanalysis, we used data from an experiment in which three out of six written words in an array were presented in auditory word-written word matching on the first three trials and all six words were presented only on the fourth trial (Forde \& Humphreys, 1995, Experiment 12). Overall, J.M. was worse on Trial 4 than on Trial 1, consistent with his performance becoming refractory over time. However, J.M. was neither more nor less likely to point to a repeated item ( 35 errors) than to point to a new item (46 errors) when he made an error on Trial 4 (binomial test, $p=$ .3). We suggest that this failure to generate neither positive perseverations nor reverse perseverations is because the representational state (either refractory or activated) spread to semantically related exemplars and created a "noisy" system in which target-competitor differentiation was difficult, with random responses often being made within the category.

\section{Implications for Models of Semantic and Lexical Memory}

To account for the contrasting patterns of refractoriness and priming across tasks that we have reported, we need to expand on different models of semantic representation. For example, in some models of semantic memory, all our knowledge about a particular item is stored in a "node," and success on tests of semantic knowledge requires activation of these nodes above some threshold level. According to this view, semantic representations about particular items are either intact or impaired, and damage at this level should result in patients showing a good degree of item-specific consistency in their performance over time. Patients should also show little effect (either beneficial or negative) from priming if the node is destroyed by brain damage. However, our data from Experiments 3 and 4 demonstrate that patients can show semantic priming effects and refractory behavior following related items when different aspects of semantic knowledge have to be retrieved (superordinate vs. more detailed item-specific knowledge, respectively). One way of accommodating this difference would be to assume that superordinate information is accessed first in a hierarchy of knowledge before detailed attribute information, and that (spared) access to the top levels of the hierarchy can be isolated from (impaired) access to the lower levels (Collins \& Loftus, 1975; Shallice, 1988; Warrington, 1975). Similarly, lexical decisions may be facilitated if they are influenced by words accessing the same superordinate semantic knowledge. This model is consistent with our data if we assume that the pattern of activation and inhibition of responses is normal at the top level of the semantic hierarchy but impaired at the lower levels representing attribute information.

In contrast, in other models of semantic memory, information is not stored in a hierarchy but in a distributed system of semantic features (e.g., Allport, 1985; Rogers et al., 2004; Tyler \& Moss, 1997). The semantic representation of a particular item (e.g., a dog) corresponds to a pattern of activity across stored semantic features (e.g., $<4$ legs $>$, $<$ tail $>,<$ head $>$, < <eyes $>$, and $<$ barks $>$ ). Some of these features would be shared with other items, and others would be more distinctive. For example, the representation of "cat" would also include the features $<4$ legs $>,<$ tail $>,<$ head $>$, and $<$ eyes $>$, but, in addition, would activate distinctive features such as $<$ meows $>$. One consequence of this is that the stimulus $d o g$ would activate a number of the semantic features for semantically related items (such as cats) but the correct target would normally be selected because the pattern of features unique to this item would reach threshold before other related items. Once a target has been selected, the activation will either dissipate or the target's representation will be actively inhibited (e.g., Dell, Burger, \& Svec, 1997). According to this account, superordinate categorizations can be made relatively quickly because a smaller subset of features needs to be activated in comparison with tasks that require identification of the item or access to finegrained information (e.g., picture-word matching, in patient J.M.). For example, activation of $<4$ legs $>$, $<$ tail $>$, $<$ head $>$, and $<$ eyes $>$ would be sufficient to determine that a dog or a cat was an animal. J.M., therefore, benefits from semantically related primes in superordinate categorization (Experiment 4) because these items activate the set of features that are common across exemplars and are useful in categorization tasks. However, for this account, it remains difficult to simultaneously explain the data on refractory effects (Experiment 3), if the semantic features are temporarily inhibited. On the other hand, if the activation of features remains abnormally high, then selection of a detailed (unique) representation of the target among related items becomes difficult. As a consequence, J.M. is impaired by semantic priming when fine-grained semantic information needs to be retrieved. This difficulty in selection may arise even when a target is repeated, because the target activates features in common with its semantic neighbors.

There is at least one other consequence of the present results for understanding the nature of our lexical and semantic knowledge - particularly the finding that there is relatively normal priming from repeated items on lexical decision even though the same repeated items behave in a refractory manner in picture-word matching (Experiment 2). This finding is consistent with there being a distinction between lexical and semantic knowledge. Prolonged activation at a lexical level facilitates lexical decision to a repeated item. In contrast, at a detailed semantic level, there is either prolonged inhibition or prolonged activation, and difficulties in selecting between semantic neighbors. The proposal that lexical knowledge can be distinguished from semantic knowledge is also supported 
by the finding that J.M.'s lexical decision was not affected by the category that words came from (Experiment 1), whereas performance on semantically mediated tasks was (e.g., on auditory word-written word matching, which we hold is semantically mediated for J.M.; see Forde \& Humphreys, 1997). This is consistent with lexical knowledge being represented independently of semantic information distinguishing between the referents of the words.

\section{The Access-Storage Debate, Priming, and Comparison Across Patient Groups}

In previous work, we have reported that J.M. showed a number of characteristics of an access impairment, according to the criteria established by Warrington and Shallice (1979; Shallice, 1988): He was inconsistent (over time) and showed an effect of rate but no effect of frequency. However, he also showed a depth-of-processing effect, since he had knowledge of superordinate semantic information even when he could not access detailed semantic knowledge; this last pattern is typically thought to occur in patients with degenerate stored knowledge (Warrington \& Shallice, 1979). Here, we have shown that J.M. also manifests facilitatory repetition and semantic priming effects when lexical and superordinate semantic information must be accessed, along with refractory behavior when detailed semantic information is required. Thus, priming effects occurred in a patient who showed patterns of performance characteristic of both access and storage disorders. Also, since the same patient manifested both facilitatory priming effects and refractory behavior, under different circumstances, we suggest that the direction of any priming effects (whether facilitatory or inhibitory) cannot be used as diagnostic of access or storage deficits in patients (see also Chertkow et al., 1989). Our results suggest that the present criteria for separating access and storage disorders are empirically incoherent (see also Humphreys \& Rumiati, 1998; Rapp \& Caramazza, 1993). However, to the best of our knowledge, J.M. is the only patient who has been tested on such a wide range of criteria. In future work, we propose that progress in distinguishing between storage and access cases can only be made by: (1) testing patients across the complete set of tests thought to distinguish between access and storage problems; (2) including independent measures that provide converging evidence on the locus of any deficit (e.g., assessing for generalization of deficits across modalities; Forde \& Humphreys, 1997); and (3) relating the data from patients to more fully articulated models of semantic memory.

\section{AUTHOR NOTE}

This work was supported by a grant awarded to E.M.E.F. from the Department of Education in Northern Ireland, and by a grant awarded to G.W.H. from the Medical Research Council. Correspondence concerning this article should be addressed to G. W. Humphreys, Behavioural and Brain Sciences Centre, School of Psychology, University of Birmingham, Birmingham B15 2TT, England (e-mail: g.w.humphreys@bham.ac.uk).

\section{REFERENCES}

Allport, D. A. (1985). Distributed memory, modular systems and dysphasia. In S. K. Newman \& R. Epstein (Eds.), Current perspectives in dysphasia. Edinburgh: Churchill Livingstone.
Blaxton, T. A., \& Bookheimer, S. Y. (1993). Retrieval inhibition in anomia. Brain \& Language, 44, 221-237.

BURTON, M. A. (1994). Learning new faces in an interactive activation and competition model. Visual Cognition, 1, 313-348.

ChertKow, H., Bub, D. N., \& Seidenberg, M. (1989). Priming and semantic memory loss in Alzheimer's disease. Brain \& Language, 36, 420-446.

Chun, M. M., \& Wolfe, J. A. (1996). Just say no: How are visual searches terminated when there is no target present? Cognitive Psychology, 30, 39-78.

Cipolotti, L., \& WarRington, E. K. (1995). Towards a unitary account of access dysphasia: A single case study. Memory, 3, 309-332.

Collins, A. M., \& Loftus, E. F. (1975). A spreading-activation theory of semantic processing. Psychological Review, 82, 407-428.

Coltheart, M. (1978). Lexical access in simple reading tasks. In G. Underwood (Ed.), Strategies of information processing (pp. 151-216). London: Academic Press.

Coltheart, M., Rastle, K., Perry, C., Langdon, R., \& Ziegler, J. (2001). DRC: A dual route cascaded model of visual word recognition and reading aloud. Psychological Review, 108, 204-256.

Dell, G. S., Burger, L. K., \& Svec, W. R. (1997). Language production and serial order: A functional analysis and a model. Psychological Review, 104, 123-147.

Durso, F. T., \& Johnson, M. K. (1979). Facilitation in naming and categorizing repeated pictures and words. Journal of Experimental Psychology: Human Learning \& Memory, 5, 449-459.

Forde, E. M. E., \& HumphreYs, G. W. (1995). Refractory semantics in global aphasia: On semantic organisation and the access-storage distinction in neuropsychology. Memory, 3, 265-307.

Forde, E. M. E., \& HuMPhreYs, G. W. (1997). A semantic locus for refractory behaviour: Implications for access-storage distinctions and the nature of semantic memory. Cognitive Neuropsychology, 14, 367-402.

Howard, D., \& Patterson, K. (1992). The Pyramids and Palm Trees Test. Bury St. Edmunds, U.K.: Thames Valley Test Company.

Humphreys, G. W., \& Rumiati, R. I. (1998). When joys come not in single spies but in battalions: Within-category and within-modality identification increases the accessibility of degraded stored knowledge. Neurocase, 4, 111-126.

JACOBY, L. L., \& DALlas, M. (1981). On the relationship between autobiographical memory and perceptual learning. Journal of Experimental Psychology: General, 110, 306-340.

Kay, J. M., Lesser, R., \& Coltheart, M. (1992). PALPA: Psycholinguistic Assessments of Language Processing in Aphasia. Hove, U.K.: Erlbaum.

McCARThY, R. A., \& KarTsounis, L. D. (2000). Wobbly words: Refractory anomia with preserved semantics. Neurocase, 6, 487-497.

McNeil, J. E., Cipolotti, L., \& Warrington, E. K. (1994). The accessibility of proper names. Neuropsychologia, 32, 193-208.

Meyer, D. E., \& SchVAneveldt, R. W. (1971). Facilitation in recognizing pairs of words: Evidence of a dependence between retrieval operations. Journal of Experimental Psychology, 90, 227-234.

Monsell, S. (1990). Frequency effects in lexical tasks: Reply to Balota and Chumbley. Journal of Experimental Psychology: General, 119, 335-339.

Morton, J. (1979). Facilitation in word recognition: Experiments causing change in the logogen model. In P. A. Kolers, M. A. Wrolstad, \& H. Bouma (Eds.), Processing of visible language. New York: Plenum.

Plaut, D. C., McClelland, J. L., Seidenberg, M. S., \& Patterson, K. (1996). Understanding normal and impaired word reading: Computational principles in quasi-regular domains. Psychological Review, 103, 56-115.

RAPP, B., \& CARAMAZZA, A. (1993). On the distinction between deficits of access and deficits of storage: A question of theory. Cognitive Neuropsychology, 10, 113-141.

RidDoch, M. J., \& Humphreys, G. W. (1993). BORB: Birmingham Object Recognition Battery. Hove, U.K.: Erlbaum.

Rogers, T. T., Lambon Ralph, M. A., Garrard, P., Bozeat, S., MCClelland, J. L., Hodges, J. R., \& Patterson, K. (2004). Structure and deterioration of semantic memory: A neuropsychological and computational investigation. Psychological Review, 111, 205-235.

Salasoo, A., Shiffrin, R. M., \& Feustel, T. C. (1985). Building permanent memory codes: Codification and repetition effects in word 
identification. Journal of Experimental Psychology: General, 114, 50-77.

SCHACTER, D. L., \& COOPER, L. A. (1993). Implicit and explicit memory for novel visual objects: Structure and function. Journal of Experimental Psychology: Learning, Memory, \& Cognition, 19, 995-1009.

Shallice, T. (1988). From neuropsychology to mental structure. Cambridge: Cambridge University Press.

SnODGRASS, J. G., \& VANDERWART, M. (1980). A standardized set of 260 pictures: Norms for name agreement, image agreement, familiarity, and visual complexity. Journal of Experimental Psychology: Human Learning \& Memory, 6, 174-215.

TyleR, L. K., \& Moss, H. E. (1997). Functional properties of concepts: Studies of normal and brain-damaged patients. Cognitive Neuropsychology, 14, 511-545.

WARRINGTON, E. K. (1975). The selective impairment of semantic memory. Quarterly Journal of Experimental Psychology, 27, 635-657.

Warrington, E. K., \& CipolotTi, L. (1996). Word comprehension: The distinction between refractory and storage impairments. Brain, 119, 611-625.

WARRINGTON, E. K., \& CRUTCH, S. J. (2004). A circumscribed refractory access disorder: A verbal semantic impairment sparing visual semantics. Cognitive Neuropsychology, 21, 299-315.

WARRINGTON, E. K., \& McCARTHY, R. [A.] (1983). Category-specific access dysphasia. Brain, 106, 859-878.

Warrington, E. K., \& McCarthy, R. A. (1987). Categories of knowledge: Further fractionations and an attempted integration. Brain, 110, 1273-1296.

Warrington, E. K., \& Shallice, T. (1979). Semantic access dyslexia. Brain, 102, 43-63.

\section{NOTES}

1. We will use the term "refractory behavior" to describe the empirical phenomenon of poorer performance on repeated items; we do not use it to describe a hypothetical mechanism for the poorer performance.

2. Statistical note: Although items were repeated in Experiment 1, we did not perform a repeated measures analysis on the data because there were so few words $(27 / 60)$ that J.M. responded to correctly on all three critical presentations [T1, T3(R), T3(N)]. To assess whether the RTs were independent from one another, we examined the correlation matrix for $\mathrm{T} 1, \mathrm{~T} 3(\mathrm{~N})$, and $\mathrm{T} 3(\mathrm{R})$. There was a significant correlation between T3(N) and T3(R) $[r(27)=.4, p=.05]$, indicating some form of dependency between RTs in these conditions (i.e., items that were responded to rapidly in $\mathrm{T} 3[\mathrm{~N}]$ were also responded to rapidly in $\mathrm{T} 3[\mathrm{R}]$ ).
Given this item dependency, we performed a one-way, repeated measures ANOVA using as many items as possible (27). This analysis replicated the significant effect of condition found in our between-items analysis $[F(2,52)=3.8, p=.03]$. Again, J.M. was faster with repeated items $(2,904 \mathrm{msec})$ than with items presented on the first trial $(3,331 \mathrm{msec})$ or with new items presented on Trial $3(4,184 \mathrm{msec})$.

In the remaining experiments in this article, we have also used the same items across conditions, but analyzed the data with between-subjects ANOVAs to keep as much data as possible. In all of the following experiments, there were no significant correlations between any of the conditions, suggesting some degree of independence in J.M.'s performance across items over time, so that a between-subjects analysis is appropriate. Note, too, that the between-subjects analyses provide a relatively conservative way to treat the data.

3. An alternative view is that the picture-word matching procedure induces some longer-term form of learning (e.g., changing the weights between representations), which might have the effect of speeding lexical decision (e.g., in Experiment 2).

APPENDIX

J.M.'s Scores for Auditory Word-Written Word Matching Across Categories

\begin{tabular}{lcclc}
\hline \multicolumn{1}{r}{ Category } & Score & & \multicolumn{1}{c}{ Category } & Score \\
Famous names & 22 & & Body parts & 18 \\
Transport & 22 & & Instruments & 17 \\
Animals & 21 & & Colors & 17 \\
Sport & 21 & & Geographical features & 17 \\
Occupations & 21 & & Kitchen items & 17 \\
Countries & 20 & & Materials & 15 \\
Cities & 20 & & Boys'names & 15 \\
Clothes & 20 & & Vegetables & 14 \\
Office items & 20 & & Furniture & 13 \\
Residences & 19 & & Units of time & 13 \\
Weather & 19 & & Parts of a room & 12 \\
Emotions & 19 & & Surnames & 11 \\
Subjects & 18 & & Girls' names & 9 \\
Fruit & 18 & & Flowers & 8 \\
\hline
\end{tabular}

Note- - Maximum correct $=24$.

(Manuscript received June 15, 2002; revision accepted for publication December 20, 2006.) 